\title{
Accommodative Load from Mobile Phone E-mail
}

\author{
Masako Omori, Masaru Miyao (Nagoya University), Hisao Ishigaki (Aichi Institute of Technology), \\ Satoshi Hasegawa (Nagoya Bunri University), Shin'ya Ishihara (Aichi University of Education), \\ Shohei Matsunuma (Telecom Express, Co., Ltd.)
}

\section{Introduction}

The number of mobile phones (MPs) in use in Japan, including PHS, had reached 8 million by the end of March 2004. The use of mobile phones has rapidly spread worldwide. Small message services (SMS) and personal digital assistants (PDA) with small displays and characters have also become popular.

These devices have small liquid crystal displays (LCDs) with poor contrast and are read at short visual distances. They are thought to cause severe visual load after long usage. Sakata et al. (2001) reported the finding that the legibility of non-backlit type game consoles is very low due to dark and low contrast screens with poor resolution.

To date, there have been few studies of visual load from accommodation of the crystalline lens to mobile phones. In the present study, we examined the visual accommodation of subjects who were gazing fixedly at e-mail texts from three different sources: high screen resolution, low screen resolution and printed paper.

\section{Materials and Methods}

\subsection{Subjects and Materials}

The subjects were 3 people aged 21 years with normal vision. The refraction of subjects' eyes is emmetropia of less than \pm 0.5 diopter (D).

In the present study, three different types of visual image were used: cellular phones with high resolution display (J-SH53) and low one (J-SH07), and printed paper. The SH07 has a low contrast ratio with poor resolution $(120 \times 160$ dots), while the SH53 has a bright display with fine resolution $(240 \times 320)$. The printed paper is clear and has no blurring (Table 1).

We measured and recorded accommodative fluctuation of three male subjects while they were viewing the e-mail text for 30 -second periods.

Visual function was tested using a custom-made apparatus. This combined an automated infrared accommodo-refractometer (Nidek AR-1100) and original binocular half-mirror system (Miyao, Otake, \& Ishihara, 1992; Miyao et al., 1996). The display images were placed in front of the small mirror for the tests. Subjects gazed at each type of image through a half (dichroic) mirror and an ordinary small mirror. The instrument objectively measured visual accommodative changes of the right eye at a $12.5 \mathrm{~Hz}$ sampling rate in both binocular and natural viewing conditions (Otake et al., 1993; Figure 1).

\subsection{Methods}

The distance between the subjects' eyes and the target on the screen was $37 \mathrm{~cm}(1.00 / 0.37=$ 2.70 diopters (D)) (Note: diopter (D) $=1 /$ distance $(\mathrm{m})$; MA (meter angle) $=1 /$ distance $(\mathrm{m})$ ). It is mean view distance for 20s (Masako et al., 2002).

The subjects were instructed to focus on a e-mail text in the each display.

We used Japanese e-mail sentences adopted randomly and shown in random order so that no subject read the same sentences.

The luminance and the contrast of the two MPs were adjusted to the appropriate levels by the tester in advance. Window blinds prevented glare or discomforts light in the lab room.

In this experiment, the sentences used contained caution and refuge information which should be accessible to people during emergencies (Sato, 2004).

One of the subjects read silently and the other 2 subjects read and uttered the sentence.

The subject started to read when the tester signaled. The subject read the displayed sentence silently several times. The accommodation became steady after 5 seconds of reading. They could read a page in about 2.5 seconds. Thus, the subjects' accommodative amplitude was recorded for $5 \mathrm{~s}$ between $10 \mathrm{~s}$ and $15 \mathrm{~s}$ while they read silently from the displayed e-mail text and averaged for the stable period for 2 seconds. 
Table 1. Three types of display used in this experiment

\begin{tabular}{llll}
\hline Target & SH07 & SH53 & Paper \\
\hline Screen resolution & $120 \times 160$ dots & $240 \times 320$ dots $\quad$ Quarter-VGA) & - \\
Color usage & 65536 & 260000 & Monochrome \\
Size & $50 \mathrm{~mm}(2 \mathrm{inch})$ & $61 \mathrm{~mm}(2.4 \mathrm{inch})$ & $64 \mathrm{~mm}(2.5 \mathrm{inch})$ \\
& $39.5(\mathrm{H}) \times 30(\mathrm{~W}) \mathrm{mm}$ & $48(\mathrm{H}) \times 37(\mathrm{~W}) \mathrm{mm}$ & $47(\mathrm{H}) \times 42(\mathrm{~W}) \mathrm{mm}$ \\
LCD type & TFT color & CG Silicon system color & - \\
\hline
\end{tabular}

Target display of the mobile phone

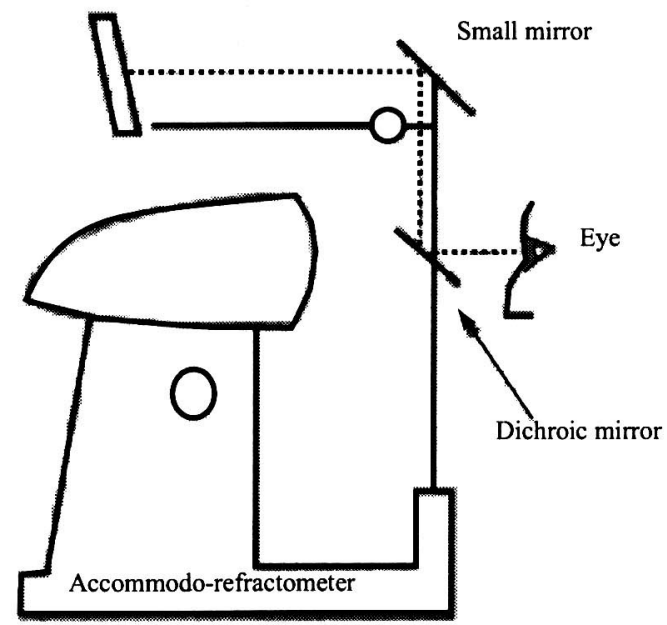

Figure 1. A schematic view of the device developed for the present experiment. The distance from eye to the target through the dichroic mirror and the small mirror is $37 \mathrm{~cm}$.

For the subjects who read and uttered the sentence, the reading time was measured. The amplitude of accommodation of the right eye while reading was measured.

The reading accommodation at the second reading was measured and averaged.

Each displayed page of text contained the same number of words per page and had approximately the same number of lines (Fig 2).

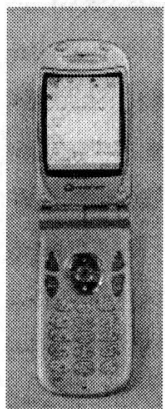

SH53

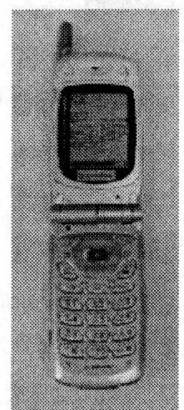

SH07
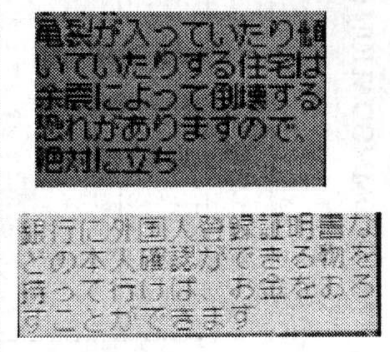

Figure 2. The cellular phones used in the experiment and the displayed sentences

\section{Results}

Table 2 shows the luminance in the dark and light areas on each screen and paper, and the contrast ratio measured by the Minolta Luminance Meter LS-100. The contrast ratios were, in the order, paper: 21.5 , SH53: 12.5 , and SH07: 2.1 .

Table 2. Mean luminance $\left(\mathrm{cd} / \mathrm{m}^{2}\right)$ in dark and light areas in each target

\begin{tabular}{llll}
\hline Target & SH07 & SH53 & Paper \\
\hline dark & 27.9 & 15.7 & 6.5 \\
light & 59.6 & 195.6 & 140.5 \\
contrast ratio & 2.1 & 12.5 & 21.5 \\
(light/dark) & & & \\
\hline
\end{tabular}

Measured by Minolta Luminance Meter LS-100

\subsection{Experiment 1: The results of voice reading}

Figure 3 shows the mean accommodative amplitude with the standard deviation for the target displayed while voice reading. In subject A, the amount of accommodation grew when looking at the SH07 (2.28 $\pm 0.27 \mathrm{D}), \mathrm{SH} 53$ (2.33 $\pm 0.46 \mathrm{D})$, and paper $(2.20 \pm 0.27 \mathrm{D})$, in that order. There was little difference among the MPs and the printed paper in mean amplitude of accommodation.

In subject $\mathrm{B}$, the amplitude of accommodation grew when he was looking at the $\mathrm{SH} 07$ was 2.06 $\pm 0.54 \mathrm{D}$, while SH53 $(1.60 \pm 0.27 \mathrm{D})$ and paper $(1.99 \pm 0.38 \mathrm{D})$ eased his accommodative tension. SH53 had weak accommodation as compared with other two, suggesting that it is easy and comfortable to focus these sentences. 


\subsection{Experiment 2: The results of silent reading}

Figure 5 shows mean accommodative amplitude with the standard deviation for the target displayed at the silent reading. In subject $\mathrm{C}$, the means of accommodative amplitude were $2.79 \pm 0.14 \mathrm{D}$ (SH07), 2.55 $\pm 0.10 \mathrm{D}$ (SH53), and $2.49 \pm 0.12 \mathrm{D}$ (a printed paper). Thus, SH07 required stronger visual accommodation than the other 2 targets.

\section{Discussion}

The contrast ratio is also an important factor for legibility of the screen. The German Industrial Standards (DIN 50535; former West Germany) called for a luminance contrast ratio of greater than 3:1 for visual objects. Also, Scharff \& Ahumada (2002) reported that the global masking index (which combines text contrast and background root mean square contrast) was a good predictor of the readability.

The relationships between dark screen and visual accommodative load have previously been studied. Miyao et al. (1993) measured and calculated the focusing speed (the 90 percent maximum amplitude of accommodation from a distant poster to the computer monitor, divided by time spent). They found that even young subjects had lower focusing speed when they looked at non-backlit dark LCDs than at other bright displays such as cathode ray tubes (CRTs) or backlit LCDs. It has been suggested that it is not easy to focus on non-backlit LCDs.

In this experiment, SH07 had the lowest contrast ratio. However in the voice reading experiment, there was little difference among the amplitude of accommodation for the three types of target in subject A.

In subject $\mathrm{B}$, easy focusing was seen on the high contrast ratio of SH53. However, there was no difference in average accommodation to paper and $\mathrm{SH} 07$, with a low contrast ratio. The visual distance in the present experiment was $37 \mathrm{~cm}$, meaning that the visual accommodative load was fairly high.

Miyao et al. (1989) suggested that a high-resolution format should be used when it is necessary to use very small characters.

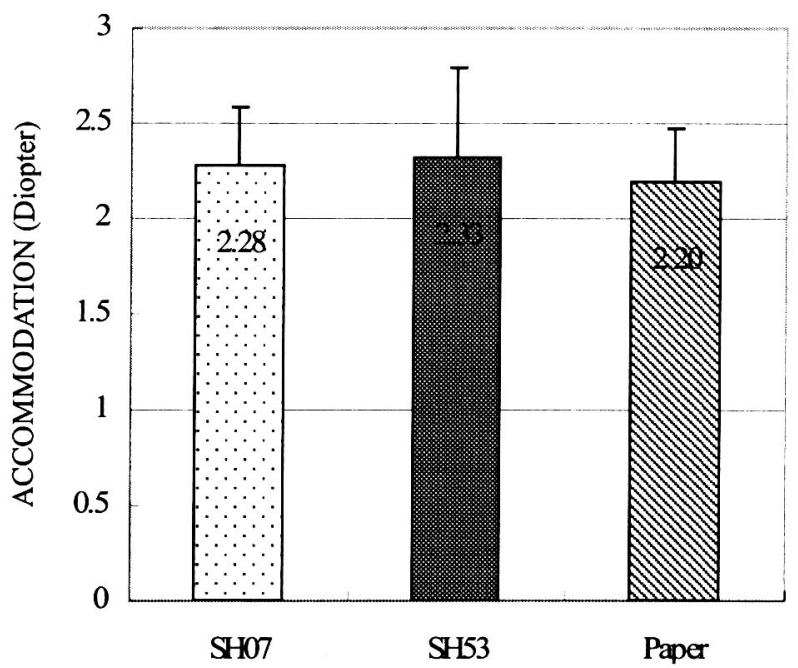

Figure 3. Mean accommodative amplitude with standard deviation for subject $\mathrm{A}$.

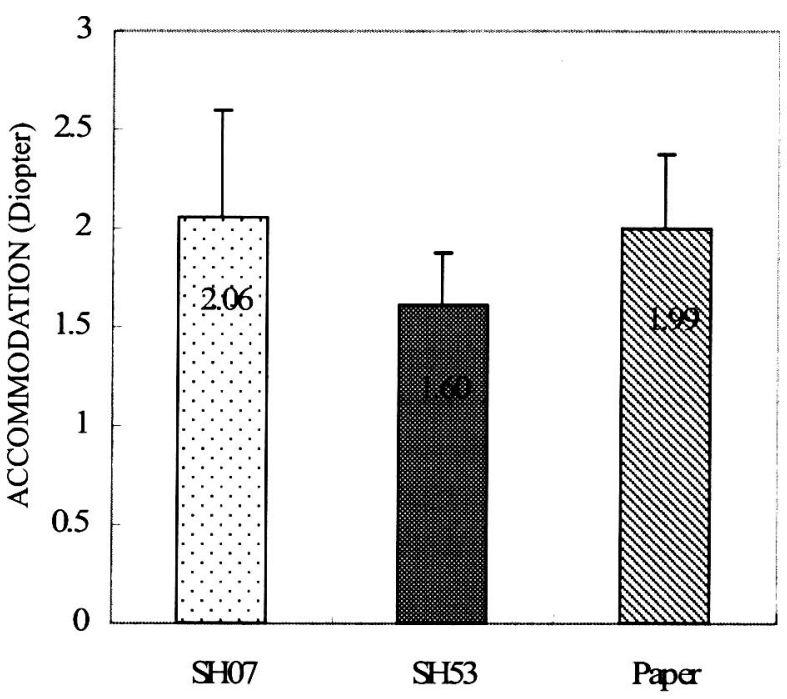

Figure 4. Mean accommodative amplitude with standard deviation for subject $B$.

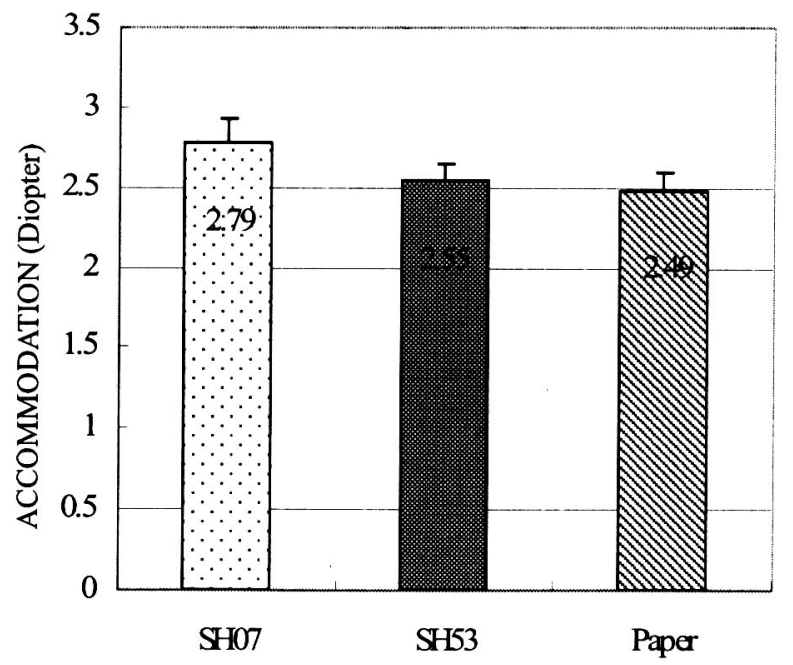

Figure 5. Mean accommodative amplitude with standard deviation for subject $\mathrm{C}$. 
ISO 9241-3 (1992) and the Japanese VDT guideline (2002) recommend that on VDTs "the minimum character height shall be 16 minutes of arc and the maximum character height shall be 24 minutes of arc for tasks in which readability is important. Character heights of 20 to 22 minutes of arc are preferred for reading tasks." Thus, character height of $3 \mathrm{~mm}$ or more is preferred, which becomes approximately $2.9 \mathrm{~mm}$ at 20 minutes of arc for a $50 \mathrm{~cm}$ visual distance. Character height of $3.7 \mathrm{~mm}$ or more is recommended for Chinese characters according to the recommended 35 minutes of arc for a 37 $\mathrm{cm}$ visual distance.

In the JIS S 0032: Guidelines for the elderly and people with disabilities--visual signs and displays--estimation of minimum legible size for Japanese single characters, the minimum legible character is presumed. According to this JIS criteria, the legible size ranges from 5.4 points to 7.3 points with a Gothic object, when people younger than 22 years of age read characters at a visual distance of $30 \mathrm{~cm}$. The number of points is converted into $\mathrm{mm}$ as follows: 5.4 points $=1.90$ $\mathrm{mm}, 7.3$ points $=2.57 \mathrm{~mm}$. These standard values (minimum legible size) were set for signs, displays or pamphlets with the dark characters and white background on boards or paper, rather than dot characters on the CRTs. In this experiment, the e-mail text used was a Gothic object and character size was $3 \times 3$ $\mathrm{mm}$. Although it was not written on paper but on an LCD, it $(3 \times 3 \mathrm{~mm})$ was larger than the minimum legible size.

It was suggested that there was little difference in average accommodative amplitude among the three displays for voice reading.

However, it appeared that the blurrier the target, the greater the accommodative amplitude for silent readings.

We may note that there were great differences in the reading speeds between voice readings and silent readings. Fast reading of small characters on the low resolution displays might cause greater amplitude of accommodation.

It is suggested that blurry, near images such as on the $\mathrm{SH} 07$ cause a greater visual accommodative load when reading e-mail in a normal manner and may lead to the development of tonic accommodation.

It was also suggested that character size needed to be $3 \mathrm{~mm}$ or more, in order to read reasonably well on low resolution and low contrast MPs.

\section{Conclusion}

The results suggested that there is little difference in average accommodative amplitude for target sentences during voice reading.

Displays with low resolution and low contrast ratio may lead to the development of tonic accommodation during silent reading of e-mail in the usual manner.

\section{References}

1) Sakata $T$, et al.: Accommodative load from handheld game consoles in kindergarten children. Environmental Health and Preventive Medicine, 6(2): 109-112, 2001.

2) Miyao M, Otake $Y, \&$ Ishihara $S: A$ newly developed device to measure objective amplitude of accommodation and papillary response in both binocular and natural viewing conditions. Jpn. J. Ind. Health, 34: 148-149, 1992.

3) Miyao M, et al.: Visual accommodation and subject performance during a stereographic object task using liquid crystal shutters. Ergonomics, 39 (11): 1294-1309, 1996.

4) Otake $Y$, et al.: An experimental study on the objective measurement of accommodative amplitude under binocular and natural viewing conditions. Tohoku J. Experimental. Medicine, 170 (2): 93-102, 1993.

5) Omori M, et al.: Visibility of mobile phones -Display characteristics and visual function-. IEA2003, 2003, 8, Seoul, Korea

6) Sato K. et al.: Multilingual and ubiquitous information system for disasters. ESK and JES Joint Symposium 2004, 5-Jun-04 7) Deutsches Institüt für Normung, DIN 5035, Beleuchtung mit künstlichem Licht, DIN Berlin, 1978.

8) Scharff LFV \& Ahumada AJ: Predicting the readability of transparent text. Journal of Vision, 2: 653-666, 2002

9) Miyao M et al.: Effects of VDT resolution on visual fatigue and readability: an eye movement approach. Ergonomics, 32 (6): 603-614, 1989.

10) ISO 9241-3.: Ergonomic requirements for office work with visual display terminals (VDTs), 1992.

11) Japan's Ministry of Health, Labour and Welfare, Guidelines for Industrial Health Controls of VDT Operations. Kihatsu No. 0405001 , April 5, 2002.

12) JIS S 0032: Guidelines for the elderly and people with disabilities-Visual signs and displays-Estimation of minimum legible size for Japanese single character, 2003. 